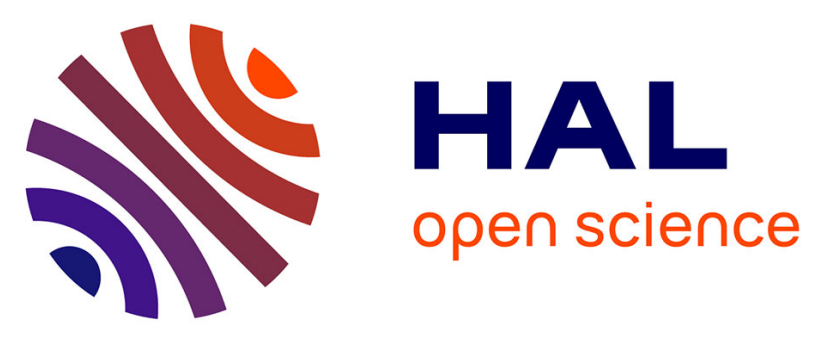

\title{
A Case Study to Identify the Drug Conjugation Site of a Site-Specific Antibody-Drug-Conjugate Using Middle-Down Mass Spectrometry
}

Oscar Hernandez-Alba, Stéphane Houel, Steve Hessmann, Stéphane Erb, David Rabuka, Romain Huguet, Jonathan Josephs, Alain Beck, Penelope Drake, Sarah Cianférani

\section{To cite this version:}

Oscar Hernandez-Alba, Stéphane Houel, Steve Hessmann, Stéphane Erb, David Rabuka, et al.. A Case Study to Identify the Drug Conjugation Site of a Site-Specific Antibody-Drug-Conjugate Using Middle-Down Mass Spectrometry. Journal of The American Society for Mass Spectrometry, 2019, 30 (11), 10.1007/s13361-019-02296-2 . hal-02341587

\section{HAL Id: hal-02341587 https://hal.science/hal-02341587}

Submitted on 8 Oct 2020

HAL is a multi-disciplinary open access archive for the deposit and dissemination of scientific research documents, whether they are published or not. The documents may come from teaching and research institutions in France or abroad, or from public or private research centers.
L'archive ouverte pluridisciplinaire HAL, est destinée au dépôt et à la diffusion de documents scientifiques de niveau recherche, publiés ou non, émanant des établissements d'enseignement et de recherche français ou étrangers, des laboratoires publics ou privés. 


\section{A case study to identify the drug conjugation site of a site-specific antibody-drug- conjugate using middle-down mass spectrometry}

Oscar Hernandez-Alba ${ }^{1}$, Stéphane Houel $^{2}$, Steve Hessmann ${ }^{1}$, Stephane Erb ${ }^{1}$, David Rabuka ${ }^{3}$, Romain Huguet ${ }^{2}$, Jonathan Josephs ${ }^{2}$, Alain Beck ${ }^{4}$, Penelope M. Drake ${ }^{3}$, and Sarah Cianférani $^{1 *}$

${ }^{1}$ Laboratoire de Spectrométrie de Masse BioOrganique, Université de Strasbourg, CNRS, IPHC UMR 7178, 67000 Strasbourg, France

${ }^{2}$ Thermo Fisher Scientific, 355 River Oaks Pkwy, San Jose, CA, 95134 United States

${ }^{3}$ Catalent Biologics West, 5703 Hollis Street, Emeryville, California 94530, United States

${ }^{4}$ IRPF, Centre d'Immunologie Pierre-Fabre (CIPF), Saint-Julien-en-Genevois, France

*Corresponding Author: Sarah CIANFERANI - sarah.cianferani@unistra.fr

Address reprint requests to: Sarah CIANFERANI - LSMBO, ECPM R5-0 - 25 Rue Becquerel - 67087 Strasbourg Cedex 2 - France - sarah.cianferani@unistra.fr 
Abstract. (fewer than 250 words)

Middle-down mass spectrometry (MD MS) has emerged as a promising alternative to classical bottom-up approaches for protein characterization. Middle level experiments after enzymatic digestion are routinely used for subunit analysis of monoclonal antibody (mAb)-related compounds, providing information on drug load distribution and average drug-to-antibody ratio (DAR). However, peptide mapping is still the gold standard for primary amino acid sequence assessment, post-translational modifications (PTM) and drug conjugation identification and localization. However, peptide mapping strategies can be challenging when dealing with more complex and heterogeneous mAb formats, like antibody-drug conjugates (ADCs).

We report here, for the first time, MD MS analysis of a third-generation site-specific DAR4 ADC using different fragmentation techniques, including higher energy collisional- (HCD), electron-transfer (ETD) dissociation and $213 \mathrm{~nm}$ ultraviolet photo-dissociation (UVPD). UVPD used as a standalone technique for ADC subunit analysis afforded, within the same liquid chromatography-MS/MS run, enhanced performance in terms of primary sequence coverage compared to HCD- or ETD-based MD approaches, and generated substantially more MS/MS fragments containing either drug conjugation or glycosylation site information, leading to confident drug/glycosylation site identification. In addition, our results highlight the complementarity of ETD and UVPD for both primary sequence validation and drug conjugation/glycosylation site assessment. Altogether, our results highlight the potential of UVPD for ADC MD MS analysis for drug conjugation/glycosylation site assessment, and indicate that MD MS strategies can improve structural characterization of empowered nextgeneration mAb-based formats, especially for PTMs and drug conjugation sites validation.

Keywords. (5 to 10 keywords): Middle-down mass spectrometry (MD MS), UVPD fragmentation, ETD, HCD, site-specific bioconjugation, antibody-drug conjugate (ADC). 


\begin{abstract}
Abbreviations.
ADC, antibody-drug conjugate; CDR, complementarity-determining region; CQA, critical quality attribute; Cys, cysteine; DAR, drug-to-antibody ratio; DARav, average drug-toantibody ratio; DLD, drug load distribution; DTT, dithiothreitol; ETD, electron transfer dissociation; Fc, fragment crystallizable; fGly, formylglycine; HCD, higher-energy collisional dissociation; IdeS, immunoglobulin degrading enzyme from Streptococcus pyogenes; IgG, immunoglobulin G; LC, light chain; LC-MS/MS, liquid chromatography-tandem mass spectrometry; mAb, monoclonal antibody; MD, middle-down; MS, mass spectrometry; PTM, post-translational modification; SMD, small molecule drug; TD, top-down; UVPD, ultraviolet photo-dissociation.
\end{abstract}




\section{INTRODUCTION}

Antibody-drug conjugates (ADCs) are a variety of monoclonal antibody (mAb)-based formats that show unmatched efficacy for treatment of many diseases, including cancers and autoimmune diseases.(1) ADCs are comprised of a mAb scaffold onto which a highly cytotoxic drug is covalently bound via a linker.(2) Among critical quality attributes (CQAs) requested by regulatory agencies such as the US Food and Drug administration or European Medicine Agency, ADC characterization requires drug load distribution (DLD) assessment, along with average drug-to-antibody ratio (DARav) determination, the amount of unconjugated mAb (D0), and residual small molecular drugs (SMDs).(3) Localization of the conjugation sites and posttranslational modifications (PTMs) is also of utmost importance. The analytical characterization of ADCs relies on a combination of state-of-the-art mass spectrometry (MS), chromatography and electrophoresis techniques. Therapeutic mAbs and their immunoconjugates are usually analyzed at different levels (intact and subunit mass analyses and peptide mapping) to achieve the best analytical characterization.

Primary sequence validation and localization of drug conjugation or PTMs are usually performed by classical peptide mapping strategies that consist of reduction, alkylation, and enzymatic (trypsin, pepsin or a combination of proteases) digestions followed by liquid chromatography-tandem mass spectrometry (LC-MS/MS) analysis of the generated peptides. Even if routinely performed, this approach has a series of limitations. First, digestion often occurs at basic $\mathrm{pH}$ and can lead to artifactual modifications of the mAb (e.g., deamidation, oxidation(4-6)). In some cases, peptide mapping approaches can also lead to incomplete sequence coverage, and thus an absence of information on the uncovered regions. Glycosylation site identification is accomplished through detection of specific glycan fragmentation patterns of the glycosylated peptide along with the presence of signature glycan oxonium ions at low mass range (204 m/z, and $366 \mathrm{~m} / \mathrm{z}) .(7-8)$ In addition, for ADCs, drug conjugation adds a 
substantial mass increase on $\mathrm{mAb}$ peptides that is combined often with a significant increase in hydrophobicity of the conjugated peptides. As a consequence, conjugated peptides are more difficult to separate during LC and are challenging for MS/MS analysis.(9-10) In addition, ADC payloads are often prone to dissociation during MS/MS in the mass spectrometer, hampering/avoiding the detection of the payload at the peptide level. So, even if well established, peptide mapping might be time consuming, labor-intensive and incomplete in terms of characterization for heterogeneous ADC formats. Thus, there is still a need for method improvement in order to provide unbiased characterization of ADC sites of conjugation and PTMs.

To overcome the limitations of MS-based bottom-up approaches, emergent alternative strategies aiming at direct sequencing of the mAb without any prior sample digestion, called top-down (TD) or its variation middle-down (MD), are appealing.(11-14) In theory, TD MS approaches combining intact mAb measurements and direct fragmentation of $150-180 \mathrm{kDa}$ protein ions should provide complete protein sequence coverage, including precise determination of PTM type and position, $\mathrm{C}$ - or N-terminus truncations and mutations. However, in practice, TD MS is still challenging and has achieved only limited success.(15) In the best cases, electron transfer dissociation (ETD)-based TD allowed for 30-35\% sequence coverage attained of a commercial $\operatorname{IgG},(12,16-17)$ due to the rigidity coming from the disulfide bridges across each IgG domain. One way to circumvent TD MS analysis shortcomings is to reduce the size of the protein. In the case of mAb-related products, this can be achieved by MD MS approaches using specific enzymes to produce $25 \mathrm{kDa}$ subunit fragments for which appropriate resolution and fragmentation techniques are available on current MS platforms (Q-TOF and Orbitrap) usually available in biopharmaceutical companies. The production of mAb subunits is often performed using IdeS (immunoglobulin-degrading enzyme of Streptococcus pyogenes)(18) digestion followed by reduction with dithiothreitol (DTT) to produce $25 \mathrm{kDa}$ 
subunits (Fc/2, LC, and Fd fragments). Optimized IdeS-based MD ETD MS/MS workflow allowed up to $\sim 50 \%$ sequence coverage to be reached for selected $\mathrm{IgG}$ fragments in a single 25 min LC run, and up to $\sim 70 \%$ when data obtained by distinct LC-MS runs are averaged.(19) Higher sequence coverages for Fc/2 and Fd fragments were even reported using IdeS-based MD ETD MS/MS on a 21 Tesla FT-ICR instrument.(11)

In the recent years, to improve TD and MD MS workflows, several groups have evaluated the possibilities of newly developed alternative ion activation techniques. Recently, Brodbelt and coworkers have demonstrated the benefits of $193 \mathrm{~nm}$ ultraviolet photo-dissociation (UVPD) and hybrid activation methods for intact protein analysis, including histone proteoforms,(20) HeLa whole-cell lysate(21) and mAb analyses.(13) Under optimal conditions, UVPD resulted in $\sim 60 \%$ overall coverage of the IgG sequence, in addition to unambiguous glycosylation site localization and extensive coverage of the antigen-binding complementarity determining regions (CDRs) in a single LC-MS/MS experiment. Combining UVPD and ETD data provided deeper sequencing and greater overall characterization of IgG subunits, highlighting potential of this new MD UVPD MS/MS strategy for the comprehensive characterization of mAb-based therapeutics.(22)

Here, we report the first MD analysis to identify the sites of conjugation and glycosylation of a site-specific ADC with drug-to-antibody ratio (DAR) of 4 (CBW-03-106) using different fragmentation techniques, including HCD, ETD and $213 \mathrm{~nm}$ UVPD. Altogether, our results highlight the potential of UVPD and the complementarity of MS/MS activation techniques from MD approaches to allow for improved primary sequence coverage and conjugation site identification of the DAR4 site-specific ADC. 


\section{MATERIAL AND METHODS}

\section{Site-specific DAR4 ADC}

CBW-03-106 was produced and modify in-house by Catalent Biologics West (CA, USA). The minimal FGE consensus sequence (CXPXR) was cloned into two specific sites of the mAb heavy chain (HC) using molecular biology technologies. The antibody is produced in a cell line with an overexpression of human FGE that oxidize the cysteine into a formyl-glycine (fGly)(23).

\section{Middle level IdeS digestion}

Site-specific DAR4 ADC was enzymatically digested using IdeS enzyme (immunoglobulindegrading enzyme of Streptococcus pyogenes) as previously described (24). Disulfide bonds were subsequently reduced during $60 \mathrm{~min}$ at $37{ }^{\circ} \mathrm{C}$ in strong denaturing conditions $(6 \mathrm{M}$ guanidine hydrochloride,) using DTT (Sigma) as a reducing agent (100 mM final DTT concentration). For MD MS analysis, the sample was infused without any previous cleaning step.

\section{Middle level LC-MS and LC-MS/MS analysis}

Separation of the Fc/2, Fd, and LC subunits were performed with a Thermo Fisher MAbPac ${ }^{\mathrm{TM}}$ RP LC column (100 mm, 1 mm i.d., 4 m particle size, and $1500 \AA$ A pore size, San Jose, CA) preheated at $60{ }^{\circ} \mathrm{C}$, using a Thermo Fisher Vanquish Horizon UHPLC system (San Jose, CA). Mobile phase A consisted of $0.1 \%$ formic acid in water, and mobile phase B was $0.1 \%$ formic acid in acetonitrile. For LC-MS/MS analysis, 1 to $1.5 \mu \mathrm{g}$ of digested ADC was loaded onto the column with $20 \%$ of $\mathrm{B}$ at a constant flow rate of $0.1 \mathrm{~mL} / \mathrm{min}$. More details about the chromatographic method can be found in the legend of Figure S5. The LC system was hyphenated to a Thermo Fisher Orbitrap Fusion Lumos Tribrid mass spectrometer (San Jose, CA) equipped with three different fragmentation modes: HCD, ETD, and $213 \mathrm{~nm}$ UVPD. For 
all experiments, the spray voltage was set to $3.6 \mathrm{kV}$ and the temperature of the ion transfer tube was $275^{\circ} \mathrm{C}$. MS/MS spectra were recorded using a mass range of 350-2000 m/z and resolving power of 120000 at $200 \mathrm{~m} / \mathrm{z}$. The multiplexing method was used and 5 precursor ions (MSX5) were selected over each specific elution window and subsequently fragmented using the three fragmentation modes. (The 5 most intense charge states were selected for HCD and UVPD. To increase the fragmentation efficiency of ETD, the highest charge states were targeted.) An isolation window of $0.8 \mathrm{~m} / \mathrm{z}$ was used for each multiplexed ions. For HCD fragmentation, ions were accelerated with $12 \mathrm{eV}$ under a constant $\mathrm{N}_{2}$ pressure of $10^{-9}$ mbar. In the case of ETD, the automatic gain control (AGC) for the precursor ions was set to $1 \times 10^{6}$ and all the precursor ions were allowed to react with the anionic fluoranthene reagent in the linear trap for $8 \mathrm{~ms}$. In order to enhance the radical-driven fragmentation, the reagent AGC was set to $7 \times 10^{5}$ with a maximum reagent injection time of $200 \mathrm{~ms}$. For UVPD MS/MS analysis, the same AGC value was used as previously specified for ETD fragmentation. Ions were resonantly activated with $213 \mathrm{~nm}$ laser during 20 or $30 \mathrm{~ms}$, delivering a total energy of 100-150 $\mu \mathrm{J}(2 \mu \mathrm{J} /$ pulse $)$.

\section{MS/MS data analysis}

MS/MS spectra were analyzed with BioPharma Finder 3.0 that includes Xtract and Prosight algorithms to perform the deconvolution and match the deconvoluted masses to the sequence, respectively. MS/MS spectra were averaged through different subunit elution windows and then deconvoluted using a $\mathrm{S} / \mathrm{N}$ of 7 . The deconvoluted masses were matched to the sequence with a $5 \mathrm{ppm}$ ion tolerance to reduce the number of false positives. Different fragment ions were considered as a function of the fragmentation technique. Thus, $b / y$ in the case of $\mathrm{HCD}, c / z$ for ETD, and $a / x, b / y$, and $c / z$ fragments were obtained in the case of the UVPD. Since the addition of the RED-106 drug molecule was performed on cysteine residues that were oxidized into formyl-glycine (fGly), all the cysteine residues contained in the $\mathrm{Fd}$, and $\mathrm{Fc} / 2$ subunits were 
considered as putative bioconjugation sites, giving rise to eight proteoforms for the $\mathrm{Fd}$, and five proteoforms for the Fc/2 (Figure S2). In the case of the Fc/2 subunit, ions corresponding to the G0F glycoform were targeted so G0F at the Asp61 was defined as a fixed modification to search the data.

\section{Bottom-up peptide mapping analysis}

Sample preparation. Fifteen micrograms of CBW-03-106 ADC was solubilized in $100 \mathrm{mM}$ ammonium acetate, $0.1 \%$ RapiGest $^{\mathrm{TM}}$ (Waters, Milford, USA) at $\mathrm{pH}$ 7.4. Disulfide reduction was performed by incubating the ADC solution with $5 \mathrm{mM}$ DTT for $30 \mathrm{~min}$ at $60{ }^{\circ} \mathrm{C}$. Alkylation was performed with $15 \mathrm{mM}$ iodoacetamide for $30 \mathrm{~min}$ in the dark. After these steps, the samples were split in two for enzymatic digestion using trypsin or pepsin.

Digestion was performed by adding trypsin (Promega, Madison, USA) to a 1:50 enzyme:substrate ratio. Samples were incubated overnight at $37^{\circ} \mathrm{C}$. The reaction was quenched by adding $1 \%$ of trifluoroacetic acid. RapiGest ${ }^{\mathrm{TM}}$ was eliminated by centrifugation at $10,000 \mathrm{~g}$ for $5 \mathrm{~min}$.

For pepsin digestion, $\mathrm{pH}$ was decreased to 2.0 prior to the addition of pepsin (Promega, Madison, USA). Digestion was performed by adding pepsin at a 1:50 enzyme:substrate ratio. Samples were incubated at $37{ }^{\circ} \mathrm{C}$ for $3 \mathrm{~h}$. The reaction was stopped by heating at $95{ }^{\circ} \mathrm{C}$ for 10 min. RapiGest ${ }^{\mathrm{TM}}$ was eliminated by a centrifugation at $10,000 \mathrm{~g}$ for $5 \mathrm{~min}$. To keep the peptides in solution, $10 \%$ of isopropanol was added after digestion.

LC-MS/MS analysis- NanoLC-MS/MS analysis was performed using a nanoAcquity UltaPerformance-LC (Waters, Milford, USA) coupled to the Q-Exactive Plus Orbitrap mass spectrometer (Thermo Scientific, Bremen, Germany) with a nanoSpray source. The peptides were trapped on a nanoACQUITY UPLC precolumn $(\mathrm{C} 18,180 \mu \mathrm{m} \times 20 \mathrm{~mm}, 5 \mu \mathrm{m}$ particle size), and then separated on a nanoACQUITY UPLC column $(\mathrm{C} 18,75 \mu \mathrm{m}$ x $250 \mathrm{~mm}$ with 1.7 
$\mu \mathrm{m}$ particle size, Waters, Milford, USA) maintained at $60^{\circ} \mathrm{C}$. Mobile phase A was $0.1 \%(\mathrm{v} / \mathrm{v})$ formic acid in water and mobile phase B was $0.1 \%(\mathrm{v} / \mathrm{v})$ formic acid in acetonitrile. A gradient (1-8\% B for $2 \min , 8-35 \%$ B for $58 \min , 35-90 \%$ B for $1 \min , 90 \%$ B for $5 \min , 90-1 \%$ B for 1 min and maintained $1 \% \mathrm{~B}$ for $20 \mathrm{~min}$ ) was used at a flow rate of $450 \mathrm{~nL} / \mathrm{min}$. The Q-Exactive Plus Orbitrap source temperature was set to $250{ }^{\circ} \mathrm{C}$ and spray voltage to $1.8 \mathrm{kV}$. Full scan MS spectra $(300-1800 \mathrm{~m} / \mathrm{z})$ were acquired in positive mode at a resolution of 140000 , a maximum injection time of $50 \mathrm{~ms}$ and an AGC target value of $3 \times 10^{6}$ charges, with lock-mass option being enabled (polysiloxane ion from ambient air at $445.12 \mathrm{~m} / \mathrm{z}$ ). The 10 most intense multiply charged peptides per full scan were isolated using a $2 \mathrm{~m} / \mathrm{z}$ window and fragmented using higher energy collisional dissociation (normalized collision energy of 27). MS/MS spectra were acquired with a resolution of 17 500, a maximum injection time of $100 \mathrm{~ms}$ and an AGC target value of $1 \times 10^{5}$, and dynamic exclusion was set to $60 \mathrm{sec}$. The system was fully controlled by XCalibur software v3.0.63, 2013 (Thermo Scientific) and NanoAcquity UPLC console v1.51.3347 (Waters).

Bottom-up data interpretation. Raw data collected were processed and converted in .mgf format. The mgf files from trypsin and pepsin digestions were merged using Mass Spectrometry Data Analysis 2.7.3 (MSDA).

The MS/MS data were interpreted using a local Mascot server with MASCOT 2.5.0 algorithm (Matrix Science, London, UK). Spectra were searched with a mass tolerance of 5 ppm for MS and 0.07 Da for MS/MS data, using none as enzyme. G0F glycosylation (+1445.45 Da), the linker modification (+652.37 Da) and the whole RED-106 payload (+1198.59 Da) were specified as variable modifications. Protein identifications were validated with Mascot ion score above 25 . Each conjugation site was manually validated based on the presence of y-ion and b-ion series and the peak intensity observed on the MS/MS spectra, using Proline 1.5 software.(25) 


\section{RESULTS}

\section{Middle level LC-MS analysis of IdeS digested CBW-03-106}

The antibody CBW-03-106 used in this study is a site-specific DAR4 ADC generated through aldehyde-specific bioconjugation.(26-32) In this case, four cysteine residues found within a specific pentapeptide consensus sequence were oxidized to formyl-glycine (fGly) with a formyl-glycine-generating enzyme.(27, 29) The fGly residues were further modified using aldehyde-specific chemistries in order to selectively conjugate four RED-106 drug molecules to the mAb structure, located on the $\mathrm{Fc} / 2$ (C213) and $\mathrm{Fd}$ (C163) subunits.(32) We first performed a middle level characterization of intact CBW-03-106 using IdeS enzymatic digestion followed by DTT reduction. An improved LC method compared to the one described in Botzanowski et al.(24) was developed and allowed separation of Fc/2, LC, and Fd ADC subunits within a 10 min run (see Material and Methods). This LC-MS analysis was used as a high resolution LC-MS survey run to provide information on subunit retention times, the charge state distribution (isotopic resolution of each peak) and subsequent sub 3 ppm mass accuracies within a single LC-MS run. Three peaks were observed corresponding to the $\mathrm{Fc} / 2$, the light chain (LC), and Fd fragments, respectively (Figure 1). Peak 1 (27307.51 $\pm 0.01 \mathrm{Da}, 2$ ppm) corresponds to the theoretical mass of the G0F glycoform of the $\mathrm{Fc} / 2$ with one molecule of RED-106; peak 2 could be unambiguously attributed to the LC of the mAb (23389.31 \pm 0.01 Da, 2 ppm), and peak 3 was assessed to the conjugated Fd subunit with one RED-106 molecule (26770.34 $\pm 0.01 \mathrm{Da}, 3 \mathrm{ppm}$ ). No signals corresponding to the unconjugated $\mathrm{Fd}$ and $\mathrm{Fc} / 2$ subunits could be detected from extracted ion chromatograms. High-resolution LC-MS analysis also revealed the presence of two common modifications on the $\mathrm{Fc} / 2$ subunit, namely $\mathrm{C}$ terminal K-clipping and N-glycosylation. 
a)

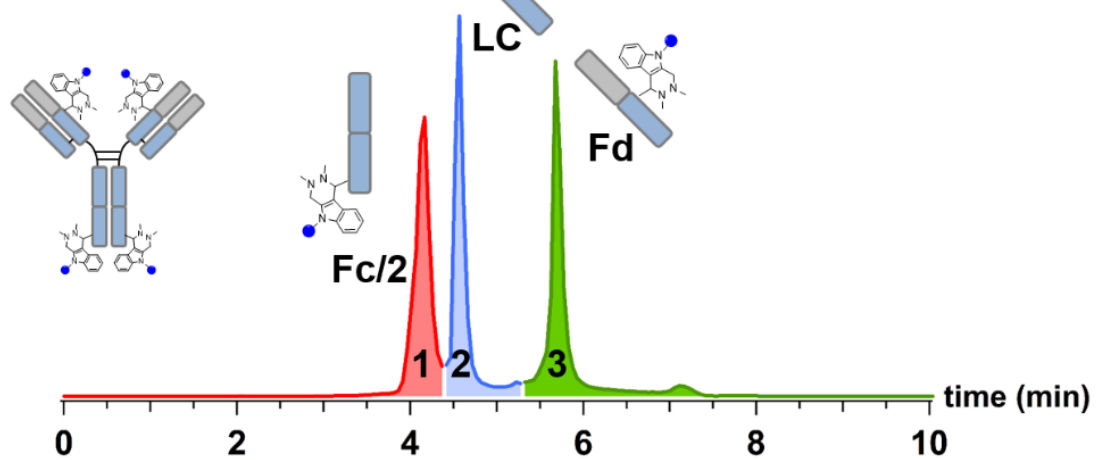

b)

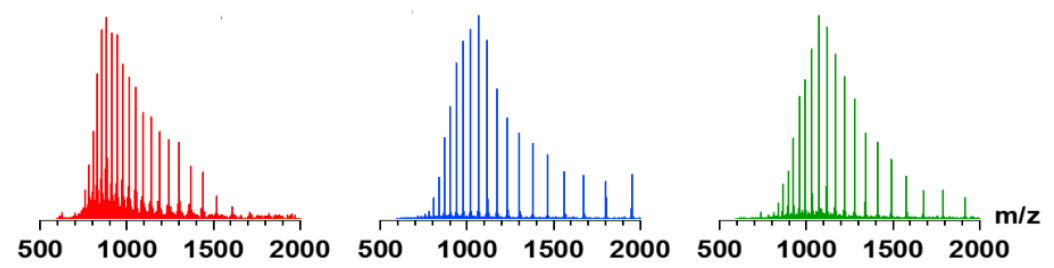

c)

\begin{tabular}{cccc} 
& Experimental mass $(\mathrm{Da})$ & Theoretical mass $(\mathrm{Da})$ & Error $(\mathrm{ppm})$ \\
\hline \hline Fc/2+1 payload & $27307.51 \pm 0.01$ & 27307.46 & 2 \\
LC & $23389.31 \pm 0.01$ & 23389.25 & 2 \\
Fd+1 payload & $26770.34 \pm 0.01$ & 26770.27 & 3
\end{tabular}

Figure 1 : Middle level LC-MS analysis of site-specific DAR4 CBW-03-106 ADC after Ides digestion and DTT reduction. Chromatogram of the IdeS-digested ADC (a). Mass spectra obtained for each subunit peak (b) and corresponding measured masses (c).

Altogether, middle level LC-MS analysis and accurate mass measurements enabled us to make conclusions about the drug localization at the subunit level: one drug was linked to the $\mathrm{Fc} / 2$ subunit and the other bound to the Fd fragment, as expected from the position of the aldehyde tags in the heavy chain mAb sequence. No signal corresponding to unbound Fd or Fc/2 was detected, leading to a DARav of $4.0 \pm 0.0$, in good agreement with previous data obtained from intact and middle level characterization.(24)

Peptide mapping for identification of drug conjugation sites of the site-specific DAR4 ADC 
Peptide mapping is the gold standard for $\mathrm{mAb}$ and $\mathrm{ADC}$ analysis to obtain optimal primary sequence coverage and to gain information on glycosylation and drug conjugation sites. In our routine mAb/ADC peptide mapping workflow, we usually combine results from two independent digestions using two complementary enzymes (trypsin and pepsin, see Material and Methods). As expected, 100\% and 99\% sequence coverages were obtained for CBW-03106 light and heavy chains (see Figure S1), respectively, which meets regulatory agencies' requirements (FDA, EMA) in terms of primary amino acid sequence verification. Peptide mapping data were next interpreted for glycosylation and drug conjugation site identifications. The first analysis using automatic workflows (including glycosylation as variable modification, see Material and Methods) for bottom-up data interpretation did not provide any information about glycosylation position. After further manual analysis, the masses measured from two precursor ions (EEQYNSTYR and TKPREEQYNSTYR) could be potentially assigned to glycopeptides bearing the G0F glycoform, but their fragmentation spectra (Figure S1c) revealed that the main fragments were obtained from the glycan fragmentation, hindering glycan peptide sequencing, and thus peptide validation by automated search tools.

For drug conjugation, C163 (Fd subunit) and C213 (Fc/2 subunit) are expected to be modified from bioconjugation reaction. No peptide bearing the intact RED-106 payload (+ 1198.59 Da) was detected. As the internal fragmentation of the ADC payload (linker-cytotoxic molecule, especially the ester bond between the linker and the drug(33)) can be induced at increasing ion internal energies, MS data were analyzed taking into account the disruption of the linkercytotoxic covalent bond (+ 652.37 Da on the conjugated amino acid). However, no peptide containing the fragmented RED-106 product could be identified. Two explanations can account for that: 1) the poor solubility of more hydrophobic drug-containing peptides, and 2) the internal dissociation of the drug-linker interaction in HCD. Further manual data interpretation using extracted ion chromatograms (XIC) of tryptic peptides ions bearing the CBW-03-106 payload 
$(1175.57 \mathrm{~m} / \mathrm{z}$ and $868.44 \mathrm{~m} / \mathrm{z})$ allowed detection of peptides. However, the most intense signal in MS/MS spectra consists of the dissociation of the drug from the conjugated peptide, resulting in an intense overwhelming signal at 547.22 and $485.22 \mathrm{~m} / \mathrm{z}$ that hinders peptide backbone fragmentation (Figure S1b). As a consequence, the conjugation site is deduced from indirect XIC data interpretation of diagnostic ions from the payload fragmentation, without specific ions of the peptide bearing the payload.

\section{MS analysis with UVPD activation affords drug conjugation site identification of the site-specific DAR4 ADC}

Since MD MS might be well suited to circumvent bottom-up limitations, we thus used UVPD in a MD MS workflow to evaluate the capabilities of this activation technique to provide fragment ions characteristic of the position of the two modifications of interest $(\mathrm{N}$-glycosylation and payload). This strategy avoids solubility issues related to hydrophobic drug-conjugated peptides and allows the use of UVPD, less prone to PTM fragmentation as compared to HCD (13, 34). Altogether, UVPD MS/MS MD experiments of the Ides digested CBW-03-106 allowed for global amino acid sequence coverage of $~ 50 \%$ (44\% Fc/2, 50\% Fd, $62 \% \mathrm{LC}$ ), as already reported for unconjugated mAbs (Figure 2).(13, 22) As the conjugation strategy targeted two cysteine residues located in the Fc/2 (C213) and Fd (C163) subunits and the Nglycoforms are also located on N61 of the heavy chain of the antibody, the discussion will mainly focus on the Fc/2 and Fd subunits. To take into account possible RED-106 internal fragmentation, the presence of the linker (without the cytotoxic drug) was included as a search parameter (see Material and Methods).

After one LC-MS/MS (20 ms UVPD) run, the sequence coverage of the Fc subunit was $44 \%$ among which 34 fragments contained the RED-106 molecule (Figure 2a). Among the 34 payload-containing fragments, $8 \mathrm{C}$-terminal fragment ions containing the $\mathrm{C} 213$ conjugation site 
can be distinguished, allowing the unambiguous identification of the $\mathrm{Fc} / 2$ conjugation site (Figure 2a). Similar to the identification of the conjugation site, UVPD activation allows for the characterization of the N-glycosylation of the CBW-03-106. The fragmentation map of the Fc/2 subunit exhibits 31 fragment ions that contain the intact G0F scaffold thus, pointing out the suitability of the UVPD fragmentation to provide efficient fragmentation of the $\mathrm{Fc} / 2$ backbone whilst preserving relative labile modifications such us glycoforms (Figure 2a).

The identification of the conjugation site on the Fd subunit is more complex owing to both the position of the conjugated cysteine in the interior regions of the $\mathrm{Fd}$ sequence (C163) and the UV fragmentation pattern (Figure 2b). In spite of the significant fragmentation yield of the Fd subunit upon UV irradiation (50\% of sequence coverage), no clear-cut evidences about the conjugation site can be afforded mostly due to the size of the fragment ions (59 amino acids average length) but also to the absence of N-terminal fragments bearing the RED-106 payload. In this case, 22 C-terminal fragment ions ( $x, y$, and $z$ ions) contain the RED-106 payload. However, all these fragments include several other cysteines that can be considered as putative conjugation sites. So the identification of the conjugation site of the Fd subunit cannot be delimited to one specific cysteine.

For these reasons, the variation of additional parameters, such as the sequence coverage and the number of payload-containing fragments, as a function of the hypothetical position of the payload conjugation site were taken into account to guide the determination of the specific conjugated cysteine. In the case of the Fd subunit, the sequence coverage increases from $37 \%$ when C22 was assumed to bear the drug to $50 \%$ for the C163-conjugated proteoform (Table S1a). In addition, $8 \mathrm{MS} / \mathrm{MS}$ fragments that contained the RED-106 molecule were observed when conjugation was supposed to be on C22 compared to $22 \mathrm{MS} / \mathrm{MS}$ fragments when C163 was hypothesized to be conjugated (Table S1a). This latter parameter (number of MS/MS fragments) remains constant when the payload is assumed on cysteine residues closer to the C- 
terminus (C202, C222, C228, and C231) while the global sequence coverage decreases (Table S1), which allowed us to reinforce the confidence that the RED-106 is more likely conjugated on the $\mathrm{C} 163$ of the Fd subunit.

a) $\mathrm{Fc} / 2$

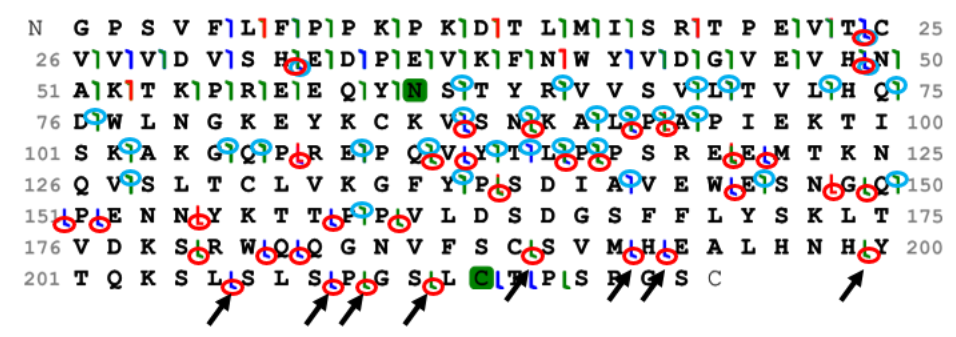

b) Fd

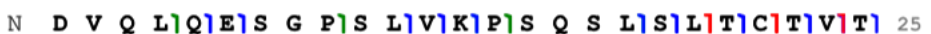

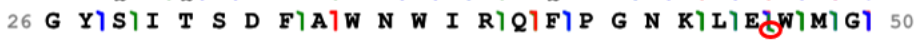

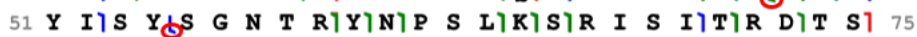

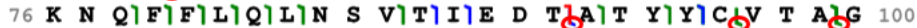

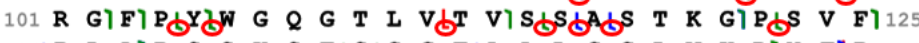

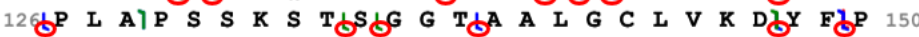

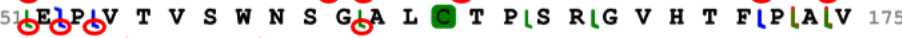

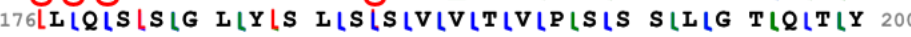
201 IILC N V N H KLI IS NLTLK V D KLK VLELPLK S C DLK T 225 226 H T C PLP CLP ALP E L L G C

c) LC

N D I L M TlQ1S P S S MlSIVISILIG D T V S I T T C H 26 SIQID IIN|S N I G W LlQ|Q|R|P|G K|S|F|K|G|LlI Y|H] 50

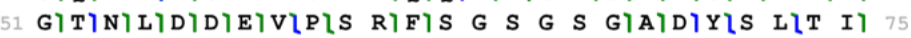

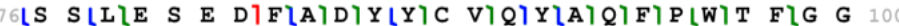
101 G T KlLlE I K RlT VlA AlPlS VlFlILFlPlPlS DlE Q L 125 126 K SLG TLALS V VlCLLlLLN N FLY]P RLELALKLV Q W K V 151LD N A LLQLS G NLSLQLELSLVLT ELQLD S KLDISLTLYLSLL 176LS S TLL TLLIS KLALDLYLELKLHLKLV YLALCLELVLTLHLQ G 200 201LLISISLPIVLTLKIS F N R G ElC C

$a / x \square b / y \square c / z$

Figure 2: Fragmentation maps of Fc/2 (a), Fd (b), and LC (c) subunits after irradiation with 213 nm UVPD when RED-106-03 payload is assumed on C213 for the Fc subunit and C163 for the Fd. The N-glycosylation (G0F) and the payload conjugation sites are highlighted with green squares. Blue and red circles show the identified cleavage sites that contain the G0F glycoform and the RED-106 payload, respectively. Specific fragment ions of C213 conjugation site are depicted with black arrows. 


\section{ETD and HCD fragmentations for more confidence in glycosylation and conjugation sites characterization}

UVPD fragmentation capabilities for CBW-03-106 characterization were benchmarked against more conventional fragmentation techniques, like $\operatorname{HCD}(35)$ and $\operatorname{ETD},(36-37)$ more frequently used in biopharmaceutical R\&D labs, to evaluate the limitations/benefits associated with each individual technique and to complement our UVPD dataset.

HCD led in fact to the lowest primary amino acid sequence coverage and the lowest number of cleavage sites that contain the RED-106 conjugation or the N-glycosylation compared to UVPD or ETD (Figure S3). In this case, HCD fragmentation yielded very low number of fragment ions characteristic of both conjugation sites ( 1 fragment ion characteristic of the $\mathrm{C} 213 \mathrm{Fc} / 2$ and 0 fragment ions corresponding to the $\mathrm{C} 163$ on the Fd subunit). In-depth analysis of MS/MS spectra showed that internal fragmentation of the RED-106 payload was favored under HCD conditions compared to UVPD (Figure S4). Intensities of diagnostic ions for payload internal fragmentation $(547.22 \mathrm{~m} / \mathrm{z}$ ) were higher with HCD than with UVPD MS/MS spectra (Figure S4). This higher payload internal fragmentation might also explain the results obtained by peptide mapping upon HCD MS/MS fragmentation (no RED-106 containing peptide detected). Additionally, the site of $\mathrm{N}$-glycosylation with $\mathrm{HCD}$ is difficult to identify, due to the low number of identified cleavage sites containing the intact G0F glycoform (Figure S3d), which corroborates peptide mapping observations.

ETD fragmentation was then carried out to induce the backbone cleavage of the site-specific DAR4 ADC subunits (Figure 3). Electron-driven fragmentation techniques have already proven their utility for mAbs sequencing at top-(12, 16-17) and middle-level(13, 22, 38) characterization. In our case, after $8 \mathrm{~ms}$ of reaction with the anionic reagent, the sequence coverage of the Fc/2, Fd, and LC subunits was 37\%, 47\%, and 52\%, respectively (Figure 3), leading to an overall $\mathrm{mAb}$ sequence coverage of $45 \%$. Albeit slightly lower sequence coverage 
was obtained compared to UVPD, overall the fragmentation maps corresponding to the ETD fragmentation exhibit fragment ions that contain the RED-106 modification. ETD generates 19 cleavage sites that contain the payload in the Fc/2 structure (Figure 3a) and four of these cleavage sites are specific of the C213 conjugation site, strengthening the results obtained with UVPD activation and thus providing more confident drug conjugation site identification. Similarly, 17 cleavage sites that contain the G0F moiety along with unambiguous localization of G0F site owing to the identification of the $c_{60}$ and $c_{61}$ consecutive fragment ions (Figure $3 \mathrm{~d}$ ) were also obtained by ETD, showing its suitability to characterize the position of the glycoforms in mAb structures. In the case of the Fd subunit, despite the detection of 16 ETD fragments bearing the RED-106 payload, no specific fragment ions of the C163 conjugation position that could led to the univocal identification of the conjugation site on C163 was observed, as previously observed upon UVPD activation (Figure 3b). While conjugation sites in the central region of the subunit sequences require the presence of $\mathrm{N}$-terminal and $\mathrm{C}$-terminal fragments specific of one single conjugation site to unambiguously determine the site of conjugation, other signature fragment ions can be used to discard the co-existence of different proteoforms. In our case, four ETD fragment ions $\left(c_{147}, c_{148}, c_{154}\right.$, and $\left.c_{157}\right)$ point out that the C143 is not conjugated, and at least 29 fragment ions support that the conjugation site is not located on cysteine amino acids at the C-terminal side (C202, C222, C228, and C231) of the Fd subunit (Figure 3b). In addition, the best sequence coverage is obtained when the RED-106 position is assumed on the C163 as previously observed using UVPD fragmentation (Table $\mathrm{S} 1 b)$. Altogether, these results lead to the indirect conclusion that the conjugation site of the Fd subunit is located on the C163. 


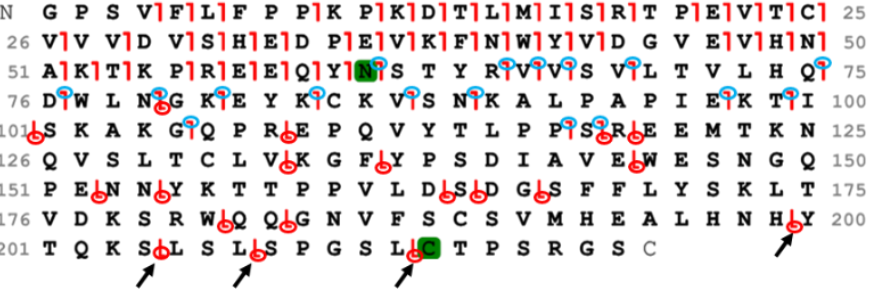

b) Fd

N D V Q L Q1E1S1G P S L V1K P1S Q1S L S L T C T V1T1 25

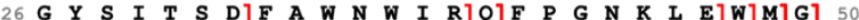
51 Y I S Y S G N T R Y N P1 S L K S R I IS1I T1R R D T S1 75

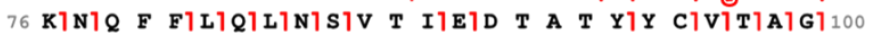

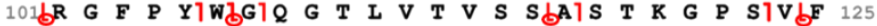

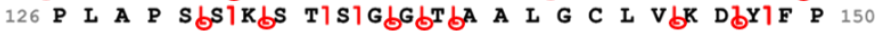
1516E P V TҺVlS W N1S G GA JL CLT PLSLRLG V HLTLF PLALV 175 176LLLQLSLSLGLLLYLSLLLSLSLVLVLTLV PLS SLSLILG T QLTLY 200

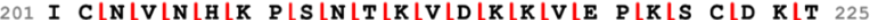
226LH T C P P C PLA P E L L G C

c) LC

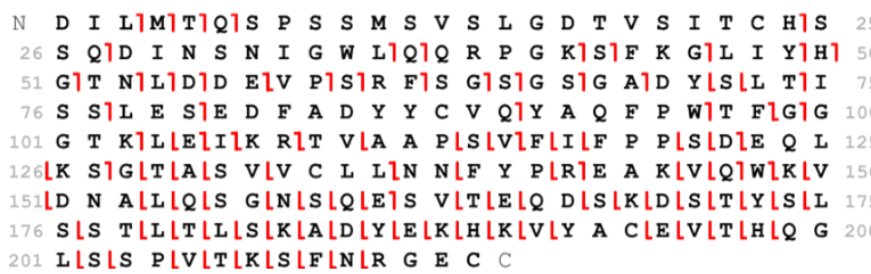

$c / 2$

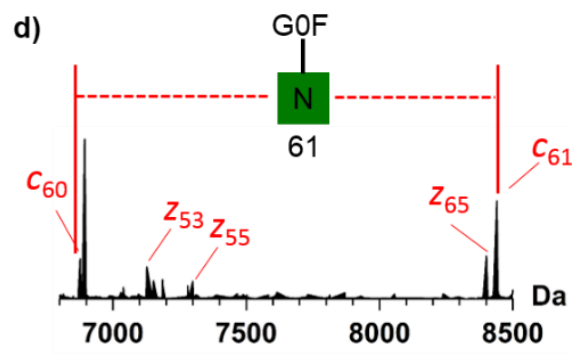

Figure 3: Fragmentation maps of Fc/2 (a), Fd (b), and LC (c) subunits after ETD fragmentation.

The N-glycosylation (G0F) and the payload conjugation sites are highlighted with green squares. Blue and red circles show the identified cleavage sites that contain the G0F glycoform and the RED-106 payload, respectively. Specific fragment ions of C213 conjugation site are depicted with black arrows. The deconvoluted ETD fragment spectrum of the Fc/2 subunit showing a mass difference between the two consecutives c60 and c61 ions that corresponds to the mass of the intact G0F structure (d).

\section{Complementarity between UVPD, HCD and ETD in MD MS of CBW-03-106}

Even though UVPD provides the best outcomes concerning the three principal parameters for ADC characterization (number of RED-106 cleavage sites, number of G0F cleavage sites and global amino acid sequence coverage), substantial benefits are obtained when MS/MS data 
from the three activation techniques are combined, highlighting the complementarity between HCD, ETD, and UVPD (Figure 4). After combining the results from the three activation techniques, the primary sequence coverage of the three ADC subunits rose to $64 \%, 74 \%$ and $83 \%$ for Fc/2, Fd and LC, respectively (Figure 4a), which approach the highest sequence coverages reported in literature using MD approaches. $(13,22)$ In addition, only a limited number of MS/MS fragments are common to the three activation modes (18 for both Fc/2 and Fd subunits, and 37 for LC), highlighting the complementarity of UVPD, HCD and ETD for overall sequence coverage (Figure S4). For CBW-03-106 primary sequence coverage using MS approaches, our results demonstrate a limited contribution of $\mathrm{HCD}$ data, but a strong complementarity between ETD and UVPD activation techniques (Figure S6), as already reported for unconjugated mAbs. $(13,22)$

When focusing on identification of the RED-106 drug conjugation (on C163 and C213) or Nglycosylation sites (N61) (Figure 4 and 5), the complementarity between UVPD, ETD, and HCD is even more obvious. Indeed, no RED-106 containing fragments are common to all three activation modes (Figure 5). Again, the number of RED-106 cleavage sites considerably increases when results of the three fragmentation techniques were combined: 58 RED-106 containing fragments can be identified within the $\mathrm{Fc} / 2$ sequence and 34 in the case of the $\mathrm{Fd}$ subunit (Figure 4b), which are significantly higher than those obtained after using only UVPD (34 and 22 respectively). Furthermore, among the total number of RED-106 containing MS/MS fragments (92), $47 \%$ result specifically from UVPD compared to $27 \%$ and $10 \%$ specific fragments generated by ETD and $\mathrm{HCD}$, respectively. It is important to notice that UVPD afforded also the highest number of payload containing fragments specific of the $\mathrm{C} 213$ on the Fc/2 subunit (8 fragments) compared to ETD (4 fragments) and HCD (1 fragment). Conversely all fragmentation techniques tested in the present work failed in direct identification of a fragment bearing RED-106 on Cys163. Altogether, the significantly higher number of drug- 
containing MS/MS fragment ions along with the number of ions characteristic of a single conjugation site obtained by UVPD compared to ETD or HCD reveals the potential of UVPD for more confident drug-conjugation site determination. However specific MS/MS fragments allowed excluding the positioning of RED-106 on cysteine's upstream from C163. Moreover, pairwise association of two fragmentation modes increased the confidence in drug conjugation binding site identification (Figure 5), through validation of cleavage sites by at least two different fragmentation techniques (3, 5 and 2 RED-106 containing MS/MS spectra of Fc/2 shared between UVPD/ETD, UVPD/HCD and ETD/HCD, respectively).
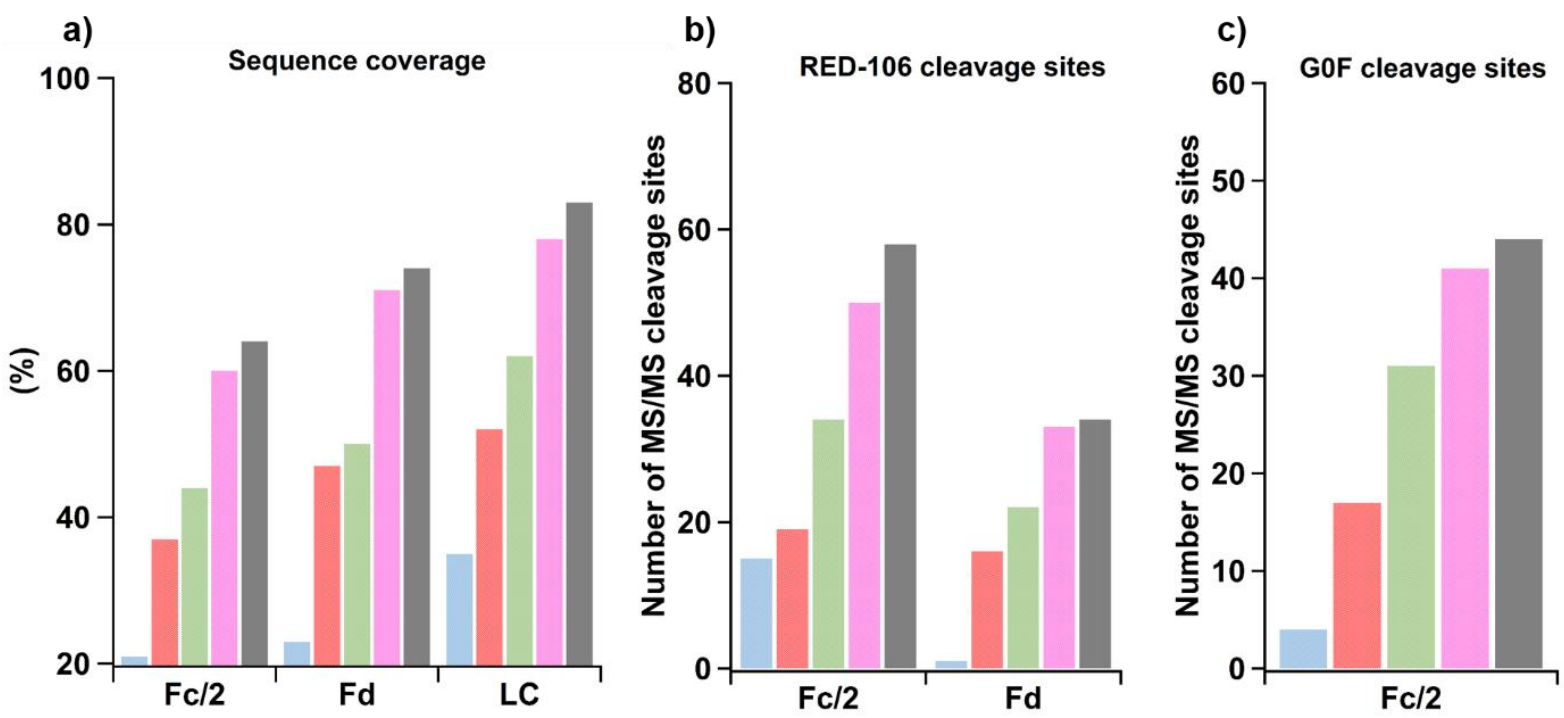

Figure 4: Contribution of HCD (blue), ETD (red), UVPD (green), ETD+UVPD (pink) and the combination of the three activation techniques (grey) to overall sequence coverage (a), number of RED-106 containing fragments (b), and number of G0F containing payloads (c).

The benefits of combining HCD, ETD, and UVPD can also be observed for the identification of the G0F glycosylation site. Thereby, 44 G0F-containing cleavage sites were identified after the combination of MS/MS data coming from the three activation techniques, while only 31 G0F containing fragments were detected using UVPD (Figure 4 and 5). In spite of the 
significantly lower number of G0F-fragments obtained upon ETD fragmentation (17) compared to UVPD (31), this activation technique allowed the identification of the glycosylation site of the CBW-03-106 at the amino acid level, in agreement with UVPD. Similarly to drug conjugation, no N61 G0F-contaning MS/MS fragment was common to the three activation techniques, illustrating the complementarity of the fragmentation techniques for G0F site identification.

Altogether, our MD results illustrate a clear complementarity between UVPD, ETD and HCD for global amino acid sequence coverage and both drug conjugation and glycosylation site identification.

Taking into account that these results imply four independent LC-MS/MS runs (one LC-MS, and three LC-MS/MS) and that HCD has only minor contribution to the MS/MS data, we evaluated the benefits of combining one LC-UVPD MS/MS and one LC-ETD MS/MS analysis. Interestingly, only minor differences can be observed in terms of sequence coverage and the number of identified payload/G0F-containing cleavage sites after combining MS/MS data from ETD and UVPD (pink bars Figure 4). For CBW-03-106 MD MS characterization, the combination of ETD and UVPD is the best option to provide the highest sequence coverage ( $\sim 65 \%)$, along with unambiguous drug conjugation and glycosylation site identification from two independent fragmentation techniques. 


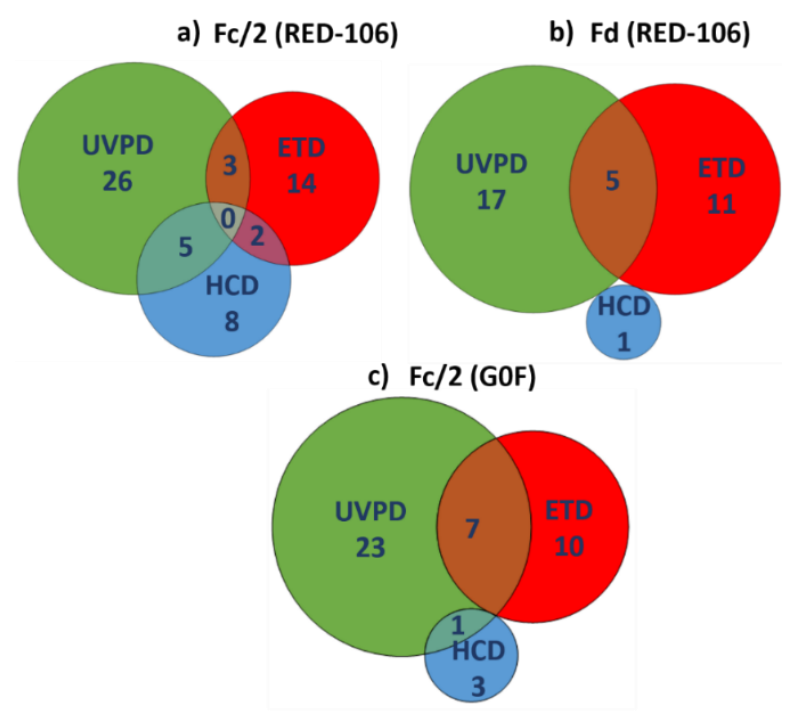

Figure 5: Venn diagram of RED-106 bearing cleavage sites obtained throughout the Fc/2 (a), and Fd (b) subunits upon UVPD, ETD, and HCD fragmentations. Number of cleavage sites containing the G0F glycoform (N61) in the Fc/2 domain after fragmentation with the three different techniques (c). 


\section{CONCLUSIONS}

We report here for the first time the use of MD approaches for the characterization of a thirdgeneration site-specific DAR4 ADC (CBW-03-106). Three fragmentation techniques (HCD, ETD, and $213 \mathrm{~nm}$ UVPD) have been used to induce the fragmentation of the Fc/2, Fd, and LC subunits of CBW-03-106. Our work highlights the great potential of UVPD, a new commercially available fragmentation technique on Orbitrap platforms, for drug conjugation and glycosylation site assessment of ADCs.

ADC characterization requires assessment of a series of CQA,(39) including primary amino acid sequence verification, glycoprofile and glycosylation site determination, DLD, DARav assessment and identification of drug conjugation sites. Currently no unique analytical workflow tackles all these issues, and multilevel analysis (intact and subunit mass analysis and peptide mapping)(39) are advised for extensive primary structure determination. Peptide mapping approaches consisting of enzymatic digestion followed by routine LC-MS/MS analysis are the gold standard for primary amino acid sequence assessment, including PTM identification and localization. Bottom-up limitations are clear in ADC drug-conjugation site identification, as many drugs increase the hydrophobicity of the resulting peptides, often leading to less soluble entities. In addition, drug-linker interactions might be dissociated in MS/MS, resulting in hardly detectable intact drug-conjugated peptides. Apart from primary acid verification, DLD, DARav, and glycoprofiles are not attainable by peptide mapping.

For all the above mentioned reasons, MD approaches have gained interest and may even complement conventional and routine bottom-up analyses when they reach their limits. Especially for ADC characterization, we demonstrate here using the CBW-03-106 site-specific DAR4 ADC that state of the art MD approaches provide rapid and accurate drug conjugation site assessment, information that was not easily obtained by peptide mapping. Advantages of middle level approaches include faster analysis time (10 min/run) combined with limited 
sample preparation. At the MS2 level, MD MS/MS analysis can provide additional specific information, like PTM (glycosylation) or drug conjugation site determination at the amino acid level. Even if peptide mapping is qualified as a critical quality test by regulatory agencies for routine amino acid sequence validation, innovative complementary approaches must be strengthened and pushed forward to improve the portfolio of analytical workflows for the more complex mAb formats being developed.

In MD approaches, UVPD used as a standalone technique showed better performance compared to HCD and ETD not only for overall sequence coverage, but also for drug or glycosylation binding site identification. Our results are in line with those obtained by the groups of Brodbelt and Kelleher on therapeutic unconjugated mAbs, $(13,22)$ suggesting that the presence of hydrophobic drugs on the amino acid scaffold does not affect UVPD performances.

A clear complementarity between UVPD and ETD in MD approaches was also demonstrated for CBW-03-106, providing complementary MS/MS information for improved sequence coverages and more accurate drug conjugation and PTM site assessment. The combination of one MS1 and two MS2 UVPD/ETD MS/MS run appears to be a good compromise for drugconjugation site assessment. Indeed, a relatively low amount of redundant information was obtained from MS/MS data from ETD and UVPD, while the overall sequence coverage and the confidence in identification of PTM/conjugation sites of the three subunits rose significantly, pinpointing a synergic effect between both activation techniques. However, identification of conjugation/PTM sites in the interior region of subunit sequences can be challenging for MD approaches since specific $\mathrm{N}$-terminal and $\mathrm{C}$-terminal fragments are needed to assign these modifications to one specific amino acid.

We believe that the routine implementation of MD approaches in R\&D laboratories will strongly depends on the development of tailored software modules based on statistical studies to correlate a specific score to each putative conjugation/PTM site. Altogether, our results 
highlight the benefits of combining MD MS analysis with multiple ion activation techniques to provide an extensive primary structure characterization of ADCs. With the ongoing progress in mass spectrometry instrumentation, activation techniques and development of adapted userfriendly software for automated scoring, validation and statistical evaluation of MS/MS spectra, we believe that MD MS will soon be mature for more routine use in biopharma companies for next-generation empowered mAb-based formats characterization.

\section{ACKNOWLEDGEMENTS}

The authors would like to thank Région Alsace for financial support in purchasing an Orbitrap Exactive Plus EMR instrument, the CNRS, the University of Strasbourg, the "Agence Nationale de la Recherche" (ANR) and the French Proteomic Infrastructure (ProFI; ANR-10-INBS-0803). O.A-H acknowledges the IdeX program of the University of Strasbourg for funding his postdoctoral fellowship. 


\section{REFERENCES}

1. Carter, P. J.,Lazar, G. A.: Next generation antibody drugs: pursuit of the 'high-hanging fruit'. Nature Reviews Drug Discovery 17 (3), 197-223 (2018).

2. Beck, A.,Goetsch, L.,Dumontet, C.,Corvaia, N.: Strategies and challenges for the next generation of antibody drug conjugates. Nature Reviews Drug Discovery 16 (5), 315-337 (2017).

3. Beck, A.,Terral, G.,Debaene, F.,Wagner-Rousset, E.,Marcoux, J.,Janin-Bussat, M. C.,Colas,

O.,Van Dorsselaer, A.,Cianferani, S.: Cutting-edge mass spectrometry methods for the multi-level structural characterization of antibody-drug conjugates. Expert Review of Proteomics 13 (2), 157-183 (2016).

4. Zhang, Z. Q.,Pan, H.,Chen, X. Y.: MASS SPECTROMETRY FOR STRUCTURAL CHARACTERIZATION OF THERAPEUTIC ANTIBODIES. Mass Spectrom. Rev. 28 (1), 147-176 (2009).

5. Ren, D.,Pipes, G. D.,Liu, D. J.,Shih, L. Y.,Nichols, A. C., Treuheit, M. J.,Brems, D. N.,Bondarenko, P. V.: An improved trypsin digestion method minimizes digestion-induced modifications on proteins. Anal. Biochem. 392 (1), 12-21 (2009).

6. Krokhin, O. V.,Antonovici, M.,Ens, W.,Wilkins, J. A.,Standing, K. G.: Deamidation of -Asn-Glysequences during sample preparation for proteomics: Consequences for MALDI and HPLC-MALDI analysis. Anal. Chem. 78 (18), 6645-6650 (2006).

7. Segu, Z. M.,Mechref, Y.: Characterizing protein glycosylation sites through higher-energy Ctrap dissociation. Rapid Commun. Mass Spectrom. 24 (9), 1217-1225 (2010).

8. Wuhrer, M.,Catalina, M. I.,Deelder, A. M.,Hokke, C. H.: Glycoproteomics based on tandem mass spectrometry of glycopeptides. J. Chromatogr. B 849 (1), 115-128 (2007).

9. Janin-Bussat, M. C.,Dillenbourg, M.,Corvaia, N.,Beck, A., Klinguer-Hamour, C.:

Characterization of antibody drug conjugate positional isomers at cysteine residues by peptide mapping LC-MS analysis. Journal of Chromatography B-Analytical Technologies in the Biomedical and Life Sciences 981 9-13 (2015).

10. Wagner-Rousset, E.,Janin-Bussat, M. C.,Colas, O.,Excoffier, M.,Ayoub, D.,Haeuw, J. F.,Rilatt, I.,Perez, M.,Corvaia, N.,Beck, A.: Antibody-drug conjugate model fast characterization by LC-MS following IdeS proteolytic digestion. Mabs 6 (1), 173-184 (2014).

11. He, L. D.,Anderson, L. C.,Barnidge, D. R.,Murray, D. L.,Hendrickson, C. L.,Marshall, A. G.: Analysis of Monoclonal Antibodies in Human Serum as a Model for Clinical Monoclonal Gammopathy by Use of 21 Tesla FT-ICR Top-Down and Middle-Down MS/MS (vol 28, pg 827, 2017). J. Am. Soc. Mass. Spectrom. 28 (5), 839-839 (2017).

12. Fornelli, L.,Ayoub, D.,Aizikov, K.,Liu, X. W.,Damoc, E.,Pevzner, P. A.,Makarov, A.,Beck, A.,Tsybin, Y. O.: Top-down analysis of immunoglobulin $G$ isotypes 1 and 2 with electron transfer dissociation on a high-field Orbitrap mass spectrometer. Journal of Proteomics 159 67-76 (2017).

13. Cotham, V. C.,Brodbelt, J. S.: Characterization of Therapeutic Monoclonal Antibodies at the Subunit-Level using Middle-Down 193 nm Ultraviolet Photodissociation. Anal. Chem. 88 (7), 40044013 (2016).

14. Tsybin, Y. O.,Fornelli, L.,Stoermer, C.,Luebeck, M.,Parra, J.,Nallet, S.,Wurm, F. M.,Hartmer, R.: Structural Analysis of Intact Monoclonal Antibodies by Electron Transfer Dissociation Mass Spectrometry. Anal. Chem. 83 (23), 8919-8927 (2011).

15. Han, X.,Jin, M.,Breuker, K.,McLafferty, F. W.: Extending Top-Down Mass Spectrometry to Proteins with Masses Greater Than 200 Kilodaltons. Science 314 (5796), 109-112 (2006).

16. Mao, Y.,Valeja, S. G.,Rouse, J. C.,Hendrickson, C. L.,Marshall, A. G.: Top-Down Structural Analysis of an Intact Monoclonal Antibody by Electron Capture Dissociation-Fourier Transform Ion Cyclotron Resonance-Mass Spectrometry. Anal. Chem. 85 (9), 4239-4246 (2013).

17. Fornelli, L.,Damoc, E.,Thomas, P. M.,Kelleher, N. L.,Aizikov, K.,Denisov, E.,Makarov, A.,Tsybin, Y. O.: Analysis of Intact Monoclonal Antibody IgG1 by Electron Transfer Dissociation Orbitrap FTMS. Molecular \& Cellular Proteomics 11 (12), 1758-1767 (2012). 
18. Chevreux, G.,Tilly, N.,Bihoreau, N.: Fast analysis of recombinant monoclonal antibodies using IdeS proteolytic digestion and electrospray mass spectrometry. Anal. Biochem. 415 (2), 212-214 (2011).

19. Fornelli, L.,Ayoub, D.,Aizikov, K.,Beck, A.,Tsybin, Y. O.: Middle-Down Analysis of Monoclonal Antibodies with Electron Transfer Dissociation Orbitrap Fourier Transform Mass Spectrometry. Anal. Chem. 86 (6), 3005-3012 (2014).

20. Greer, S. M.,Brodbelt, J. S.: Top-Down Characterization of Heavily Modified Histones Using 193 nm Ultraviolet Photodissociation Mass Spectrometry. Journal of Proteome Research 17 (3), 1138-1145 (2018).

21. Cleland, T. P.,DeHart, C. J.,Fellers, R. T.,VanNispen, A. J.,Greer, J. B.,Leduc, R. D.,Parker, W. R.,Thomas, P. M.,Kelleher, N. L.,Brodbelt, J. S.: High-Throughput Analysis of Intact Human Proteins Using UVPD and HCD on an Orbitrap Mass Spectrometer. Journal of Proteome Research 16 (5), 20722079 (2017).

22. Fornelli, L.,Srzentić, K.,Huguet, R.,Mullen, C.,Sharma, S.,Zabrouskov, V.,Fellers, R. T.,Durbin, K. R.,Compton, P. D.,Kelleher, N. L.: Accurate Sequence Analysis of a Monoclonal Antibody by Top-Down and Middle-Down Orbitrap Mass Spectrometry Applying Multiple Ion Activation Techniques. Anal. Chem. (2018).

23. Barfield, R. M.,Rabuka, D., Leveraging Formylglycine-Generating Enzyme for Production of Site-Specifically Modified Bioconjugates. In Noncanonical Amino Acids: Methods and Protocols, Lemke, E. A., Ed. Springer New York: New York, NY, 2018; pp 3-16.

24. Botzanowski, T.,Erb, S.,Hernandez-Alba, O.,Ehkirch, A.,Colas, O.,Wagner-Rousset, E.,Rabuka, D.,Beck, A.,Drake, P. M.,Cianférani, S.: Insights from native mass spectrometry approaches for topand middle- level characterization of site-specific antibody-drug conjugates. mAbs 9 (5), 801-811 (2017).

25. Carapito, C.,Lane, L.,Benama, M.,Opsomer, A.,Mouton-Barbosa, E.,Garrigues, L.,de Peredo, A. G.,Burel, A.,Bruley, C.,Gateau, A.,Bouyssie, D.,Jaquinod, M.,Cianferani, S.,Burlet-Schiltz, O.,Van Dorsselaer, A.,Garin, J.,Vandenbrouck, Y.: Computational and Mass-Spectrometry-Based Workflow for the Discovery and Validation of Missing Human Proteins: Application to Chromosomes 2 and 14 Journal of Proteome Research 14 (9), 3621-3634 (2015).

26. Drake, P. M.,Carlson, A.,McFarland, J. M.,Bañas, S.,Barfield, R. M.,Zmolek, W.,Kim, Y. C.,Huang, B. C. B.,Kudirka, R.,Rabuka, D.: CAT-02-106, a Site-Specifically Conjugated Anti-CD22 Antibody Bearing an MDR1-Resistant Maytansine Payload Yields Excellent Efficacy and Safety in Preclinical Models. Molecular Cancer Therapeutics 17 (1), 161-168 (2018).

27. York, D.,Baker, J.,Holder, P. G.,Jones, L. C.,Drake, P. M.,Barfield, R. M.,Bleck, G. T.,Rabuka, D.: Generating aldehyde-tagged antibodies with high titers and high formylglycine yields by supplementing culture media with copper(II). BMC Biotechnology 16 (1), 23 (2016).

28. Kudirka, R. A.,Barfield, R. M.,McFarland, J. M.,Drake, P. M.,Carlson, A., Bañas, S.,Zmolek, W.,Garofalo, A. W.,Rabuka, D.: Site-Specific Tandem Knoevenagel Condensation-Michael Addition To Generate Antibody-Drug Conjugates. ACS Medicinal Chemistry Letters 7 (11), 994-998 (2016).

29. Kudirka, R.,Barfield, Robyn M.,McFarland, J.,Albers, Aaron E.,de Hart, Gregory W.,Drake, Penelope M.,Holder, Patrick G.,Banas, S.,Jones, Lesley C.,Garofalo, Albert W.,Rabuka, D.: Generating Site-Specifically Modified Proteins via a Versatile and Stable Nucleophilic Carbon Ligation. Chemistry \& Biology 22 (2), 293-298 (2015).

30. Holder, P. G.,Jones, L. C.,Drake, P. M.,Barfield, R. M.,Bañas, S., de Hart, G. W.,Baker, J.,Rabuka, D.: Reconstitution of Formylglycine-generating Enzyme with Copper(II) for Aldehyde Tag Conversion. The Journal of Biological Chemistry 290 (25), 15730-15745 (2015).

31. Agarwal, P.,Kudirka, R.,Albers, A. E.,Barfield, R. M.,de Hart, G. W.,Drake, P. M.,Jones, L. C.,Rabuka, D.: Hydrazino-Pictet-Spengler Ligation as a Biocompatible Method for the Generation of Stable Protein Conjugates. Bioconjugate Chem. 24 (6), 846-851 (2013).

32. Rabuka, D.,Rush, J. S.,deHart, G. W.,Wu, P.,Bertozzi, C. R.: Site-specific chemical protein conjugation using genetically encoded aldehyde tags. Nature Protocols 71052 (2012). 
33. Sang, H.,Lu, G.,Liu, Y.,Hu, Q.,Xing, W.,Cui, D.,Zhou, F.,Zhang, J.,Hao, H.,Wang, G.,Ye, H.: Conjugation site analysis of antibody-drug-conjugates (ADCs) by signature ion fingerprinting and normalized area quantitation approach using nano-liquid chromatography coupled to high resolution mass spectrometry. Anal. Chim. Acta 955 67-78 (2017).

34. Halim, M. A.,MacAleese, L.,Lemoine, J.,Antoine, R.,Dugourd, P.,Girod, M.: Ultraviolet, Infrared, and High-Low Energy Photodissociation of Post-Translationally Modified Peptides. J. Am. Soc. Mass. Spectrom. 29 (2), 270-283 (2018).

35. Olsen, J. V.,Macek, B.,Lange, O.,Makarov, A.,Horning, S.,Mann, M.: Higher-energy C-trap dissociation for peptide modification analysis. Nature Methods 4709 (2007).

36. Coon, J. J.,Ueberheide, B.,Syka, J. E. P.,Dryhurst, D. D.,Ausio, J.,Shabanowitz, J.,Hunt, D. F.:

Protein identification using sequential ion/ion reactions and tandem mass spectrometry. Proceedings of the National Academy of Sciences of the United States of America 102 (27), 9463-9468 (2005).

37. Syka, J. E. P.,Coon, J. J.,Schroeder, M. J.,Shabanowitz, J.,Hunt, D. F.: Peptide and protein sequence analysis by electron transfer dissociation mass spectrometry. Proceedings of the National Academy of Sciences of the United States of America 101 (26), 9528-9533 (2004).

38. Srzentic, K.,Nagornov, K. O.,Fornelli, L.,Lobas, A. A.,Ayoub, D.,Kozhinov, A. N.,Gasilova, N.,Menin, L.,Beck, A.,Gorshkov, M. V.,Aizikov, K.,Tsybin, Y. O.: Multiplexed Middle-Down Mass Spectrometry as a Method for Revealing Light and Heavy Chain Connectivity in a Monoclonal Antibody. Anal. Chem. 90 (21), 12527-12535 (2018).

39. Beck, A.,Terral, G.,Debaene, F.,Wagner-Rousset, E.,Marcoux, J.,Janin-Bussat, M.-C.,Colas, O.,Dorsselaer, A. V.,Cianférani, S.: Cutting-edge mass spectrometry methods for the multi-level structural characterization of antibody-drug conjugates. Expert Review of Proteomics 13 (2), 157-183 (2016). 


\section{LEGENDS FOR FIGURES AND TABLES}

Figure 1: Middle level LC-MS analysis of site-specific DAR4 CBW-03-106 ADC after Ides digestion and DTT reduction. Chromatogram of the IdeS-digested ADC (a). Mass spectra obtained for each subunit peak (b) and corresponding measured masses (c).

Figure 2: Fragmentation maps of Fc/2 (a), Fd (b), and LC (c) subunits after irradiation with 213 nm UVPD when RED-106-03 payload is assumed on C213 for the Fc subunit and C163 for the Fd. The N-glycosylation (G0F) and the payload conjugation sites are highlighted with green squares. Blue and red circles show the identified cleavage sites that contain the G0F glycoform and the RED-106 payload, respectively. Specific fragment ions of C213 conjugation site are depicted with black arrows.

Figure 3: Fragmentation maps of Fc/2 (a), Fd (b), and LC (c) subunits after ETD fragmentation. The N-glycosylation $(\mathrm{G} 0 \mathrm{~F})$ and the payload conjugation sites are highlighted with green squares. Blue and red circles show the identified cleavage sites that contain the G0F glycoform and the RED-106 payload, respectively. Specific fragment ions of C213 conjugation site are depicted with black arrows. The deconvoluted ETD fragment spectrum of the $\mathrm{Fc} / 2$ subunit showing a mass difference between the two consecutives c60 and c61 ions that corresponds to the mass of the intact G0F structure (d).

Figure 4: Contribution of HCD (blue), ETD (red), UVPD (green), ETD+UVPD (pink) and the combination of the three activation techniques (grey) to overall sequence coverage (a), number of RED-106 containing fragments (b), and number of G0F containing payloads (c). 
Figure 5: Venn diagram of RED-106 bearing cleavage sites obtained throughout the Fc/2 (a), and Fd (b) subunits upon UVPD, ETD, and HCD fragmentations. Number of cleavage sites containing the G0F glycoform (N61) in the Fc/2 domain after fragmentation with the three different techniques (c). 